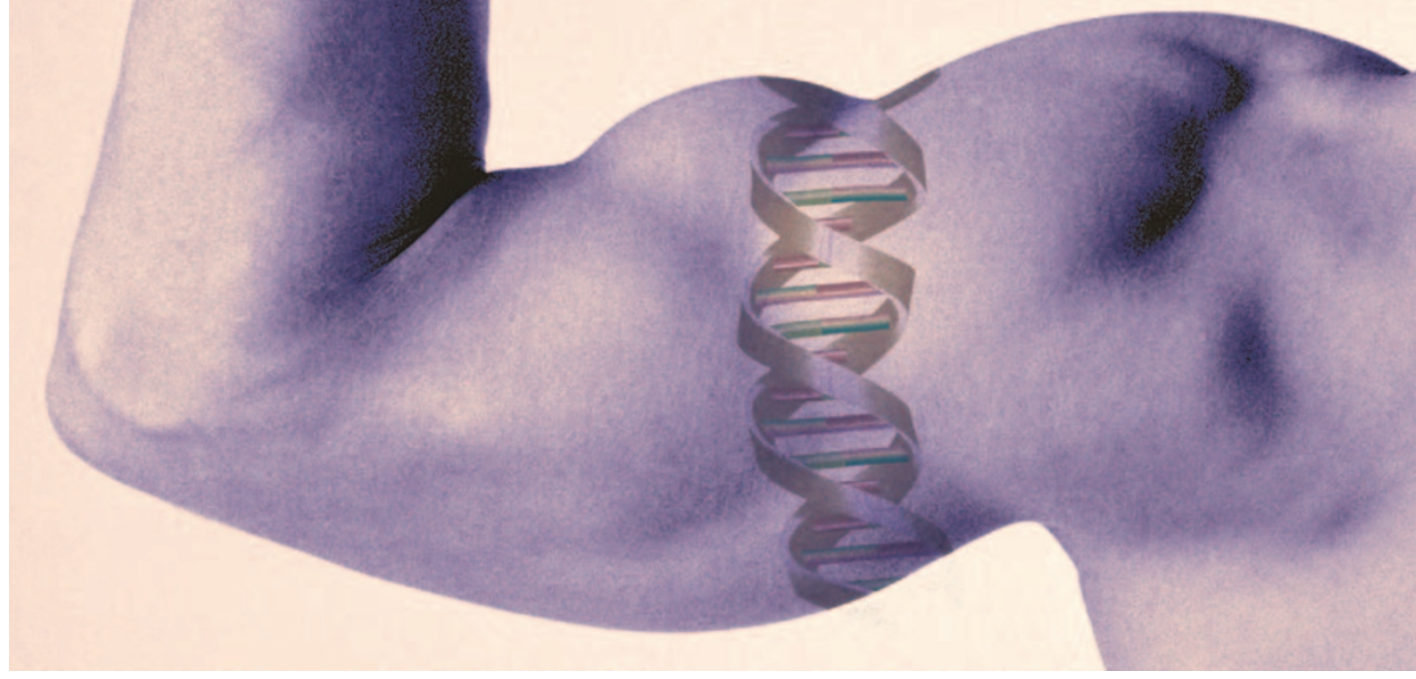

\title{
Tattoos that your mother will like
}

Immune responses that are elicited following the administration of DNA vaccines tend to develop rather slowly, and to achieve these responses, the vaccines need to be administered at several time points. Now, Adriaan Bins and colleagues report a new vaccination strategy that results in potent antibody and T-cell responses within 12 days.

It is thought that the slow development of immune responses after DNA vaccination is a consequence of only a small number of cells being transfected and of these cells only expressing a small amount of antigen, although there is little direct evidence for this. So, the authors set out to address this question directly. Initially, they showed that administration of a DNA vaccine using a tattoo device - which delivers DNA to the upper skin layers and targets a large surface area - resulted in T-cell responses. Antigen-specific T-cell responses were detected using peptide-MHC-class-I tetramers after delivery of the DNA 3 times at 2-week intervals.

Next, the authors monitored antigen-expression kinetics after delivery of DNA either by the tattooing method or intramuscularly. The DNA vaccine that was used consisted of a plasmid encoding part of influenza-A-virus nucleoprotein fused to fire-fly luciferase; after a single dose of vaccine, administration of the substrate luciferin to anaesthetized mice allowed antigen expression to be quantified. Intramuscular injection resulted in a high level of antigen expression that peaked at 1 week and remained high for 4 weeks. By contrast, intradermal delivery by the tattoo method resulted in one-tenth of the maximum antigen-expression level achieved by intramuscular injection, and this peaked at 6 hours and then diminished in the next 4 days.

So, how do these antigen-expression kinetics relate to immune responses? To address this, the authors assessed antigen presentation to naive $\mathrm{T}$ cells. They found that, although intramuscular injection resulted in a higher level of antigen expression, presentation to naive $\mathrm{T}$ cells was more efficient after delivery by intradermal tattoo.

On the basis of these data, the authors reasoned that reducing the standard 2-week interval between vaccinations might lead to faster induction of $\mathrm{T}$-cell responses following delivery of DNA by tattoo. They then tested a new protocol, in which DNA was delivered 3 times at 3-day intervals. This strategy resulted in significant $\mathrm{T}$-cell responses (with $4-8 \%$ of the total CD8 ${ }^{+} \mathrm{T}$-cell population specific for the antigen) at $\mathbf{1 2}$ days after the initial dose. To determine whether this was a consequence of increased absolute amounts of antigen expression or of increased duration of expression, they compared the delivery of three consecutive doses with the delivery of a single dose that contained threefold more DNA. They found that only the multiple applications had any effect; therefore, it is prolonged antigen expression that is the key feature.

The authors then assessed the efficacy of this strategy in a physiological situation. Short-interval tattoo application of a plasmid encoding residues 49-77 of the human papillomavirus (HPV) protein E7 resulted in rejection of established tumours in the HPV E6/E7-transformed TC-1 tumour-cell model, and this coincided with the onset of antigen-specific $\mathrm{CD}^{+} \mathrm{T}$-cell responses (which comprised up to $15 \%$ of the $\mathrm{CD8}^{+} \mathrm{T}$-cell population). This strategy also resulted in protection against infection with influenza A virus, and this protection corresponded with the induction of neutralizing antibodies.

This study establishes a strategy for short-interval DNA vaccinations that results in antibody and $\mathrm{T}$-cell responses within 12 days in mice. Further work will be required to establish the efficacy of this straightforward and inexpensive strategy in humans.

Elaine Bell

(2) References and links

ORIGINAL RESEARCH PAPER Bins, A. D. et al. A rapid and potent DNA vaccination strategy defined by in vivo monitoring of antigen expression. Nature Med. 19 Jun 2005 (doi:10.1038/nm1264)

\section{IN THE NEWS}

Colour-coded bacterium A study undertaken by scientists at the University of California, San Diego (UCSD) and published in The Journal of Experimental Medicine indicates that the pigment responsible for the characteristic golden colour of Staphylococcus aureus is a virulence factor that enables $S$. aureus to neutralize neutrophil host-defence mechanisms.

S. aureus can be a deadly human pathogen; it flourishes in hospitals, causing infections of the bloodstream and surgical wounds. The emergence of antibioticresistant strains, known as methicillin-resistant $S$. aureus (MRSA), is a major threat to public health and means that alternative treatment strategies are being sought. S. aureus produces carotenoids, which give it its characteristic golden colour. These molecules are similar to those that make carrots orange, which are known to have anti-oxidant properties. The authors showed that a mutant strain of $S$. aureus that could not produce carotenoids was more susceptible to being killed by reactive-oxygen species produced by neutrophils than was wild-type $S$. aureus, and this mutant did not form abscesses when injected into the skin of mice.

The senior author of the paper, Victor Nizet, said that the study "provides a novel target for treatment of serious Staph infections" (UCSD News). He suggests that "a treatment strategy to inhibit the Staph pigment would disarm the pathogen, making it susceptible to clearance by our normal immune defenses." (UCSD News). However, Mark Enright, from the University of Bath (UK), said that, although the research raised hope for developing new ways to combat

S. aureus, "Staph has a large number of virulence factors and toxins and to target just one of these may prove inadequate." (BBC News).

Karen Honey 